\title{
Well-defined quantum soliton masses without supersymmetry
}

\author{
Jarah Evslin (1) \\ Institute of Modern Physics, NanChangLu 509, Lanzhou 730000, China \\ and University of the Chinese Academy of Sciences, \\ YuQuanLu 19A, Beijing 100049, China
}

(Received 2 December 2019; accepted 27 February 2020; published 16 March 2020)

\begin{abstract}
Twenty-two years ago, Rebhan and van Nieuwenhuizen showed that loop corrections to the mass of a quantum soliton depend on a choice of matching condition for the regulators of the vacuum and one-soliton sector Hamiltonians. In supersymmetric theories, regulators which preserve supersymmetry yield the correct quantum corrections, as these are dictated by supersymmetry. However, in a general theory. it is not known which matching condition yields the correct mass. We use the leading term in the operator that creates the soliton to construct the regulated one-soliton sector Hamiltonian from that of the vacuum sector, providing the correct matching condition. As an application, we derive a simple formula for the one-loop mass of a kink in a large class of $(1+1)$-dimensional scalar field theories, and also, at one loop, we diagonalize the Hamiltonian which describes the kink excitations.
\end{abstract}

DOI: 10.1103/PhysRevD.101.065005

Quantum solitons play a critical role in many fields, from the vortices inside of superconductors and neutron star superfluids to the monopoles or center vortices that may be responsible for confinement in quantum chromodynamics to the branes whose identification sparked the second superstring revolution. In the semiclassical regime, the mass of a quantum soliton can be calculated in a loop expansion. This expansion requires both the vacuum sector Hamiltonian $H$ and the one-soliton sector Hamiltonian $H_{K}$ to be simultaneously regularized and renormalized. The regulators must then be taken to infinity. However, in Ref. [1] it was shown that the relationship between the regulators when they are taken to infinity affects the answer, with some prescriptions yielding the correct quantum mass and some yielding incorrect masses.

In this paper, we argue that the following prescription yields the correct matching condition. Let $\mathcal{D}_{f}$ be the unitary operator which shifts the values of the fields from a vacuum value to the classical soliton solution. Then the two regularized Hamiltonians are related by the similarity transformation

$$
H_{K}=\mathcal{D}_{f}^{-1} H \mathcal{D}_{f}
$$

This definition completely specifies the regularized $H_{K}$ given the regularized $H$. Within the validity of the

Published by the American Physical Society under the terms of the Creative Commons Attribution 4.0 International license. Further distribution of this work must maintain attribution to the author(s) and the published article's title, journal citation, and DOI. Funded by SCOAP ${ }^{3}$. semiclassical expansion, the soliton masses can now be computed perturbatively using $H_{K}$. We perform this exercise for a broad class of kink solutions, recovering a general expression for the mass at one loop which agrees with that of Ref. [2] for the $\phi^{4}$ kink and [3] for the sine-Gordon soliton.

The mass of a soliton in a quantum field theory is the difference between the lowest energies $E_{K}$ and $E_{0}$ of states in the one-soliton and vacuum sectors, respectively. In a quantum field theory, both of these numbers are generally infinite. The infrared divergences that arise from constant density at an infinite volume are easily treated, for example, by adding a constant to the Hamiltonian density. More troublesome are the ultraviolet divergences. The standard approach to these [2-4] is to separately regularize both the soliton and vacuum sectors with regulators parametrized by $r_{K}$ and $r_{0}$, respectively, and then add counterterms to each of which are fixed using a renormalization condition. The soliton mass is thus

$$
M_{s}=\lim _{r_{K}, r_{0} \rightarrow \infty} E_{K}^{r_{K}}-E_{0}^{r_{0}}+\mathrm{ct}
$$

where regulator-dependent counterterms ct have been included and subtracted in the soliton and vacuum sectors, respectively.

Equation (2) is quite ill defined. While the counterterms can be and are chosen to eliminate ultraviolet divergences, the remaining finite contributions depend on the relation between $r_{0}$ and $r_{K}$ when they are both taken to infinity. The simplest regularization schemes correspond to removing certain ultraviolet modes, and so this relation between $r_{0}$ and $r_{K}$ corresponds to an identification of cutoffs in the 
vacuum and soliton sectors. There are many choices of identification. For example, in the case of the $\phi^{4}$ kink in $1+1$ dimensions, Ref. [2] compactified their theory so that their spectra were discrete and identified modes by pairing them in order of increasing energy. The motivation for our work comes from Ref. [1], which showed that matching based on mode counting yields a different finite contribution to the mass than a matching based on a fixed UV energy cutoff.

The fact that the expression (2) depends on the matching prescription already at one loop led researchers to wonder which matching prescription, if any, is correct. More prescriptions were invented [5-7], and the results were checked against expectations from integrability and supersymmetry. In Ref. [5], the authors proposed a general definition of the kink mass in the special case of a symmetric potential. It requires a compactification of size $L$ as well as a massless limit, and it is suggested that a double scaling limit of $L$ and the mass exists in which finite mass effects do not affect the results. In Ref. [8], a distant antisoliton was added, and the author argues that as a result the boundary conditions with the pair are the same as the vacuum sector and so the ambiguity should vanish. Once the dust settled, it was concluded [4] that some matching prescriptions provide the expected results from integrability and supersymmetry at one loop, while others are "bad" [9]. In the supersymmetric case, it was possible to obtain the correct mass corrections by using a regulator that preserves the supersymmetry [10]. However, it remained unclear whether the successful prescriptions continue to produce the correct answer in the absence of integrability or supersymmetry or, more generally, beyond one loop.

In this paper, we will use an operator which takes the vacuum sector to the one-kink sector to derive the correct matching prescription, which can then be used reliably to calculate the mass of a kink to any order in perturbation theory. As an application, we will calculate the mass of a kink in a fairly general class of scalar theories at one loop. As an illustration, the case of the kink in the $\phi^{4}$ theory is worked out in gory detail in the companion paper [11].

We will consider a $(1+1)$-dimensional theory of a scalar field $\phi(x)$ with Hamiltonian

$$
\begin{aligned}
H & =H_{0}+H_{I}, \quad H_{I}=\int d x:\left[\frac{M^{2}}{g^{2}} V(g \phi(x))\right]:, \\
H_{0} & =\int d x:\left[\frac{1}{2} \pi(x) \pi(x)+\frac{1}{2} \partial_{x} \phi(x) \partial_{x} \phi(x)\right]:
\end{aligned}
$$

where $\pi(x)$ is the conjugate momentum to $\phi(x)$ and the normal ordering will be defined momentarily. The constant $g$ has units of action ${ }^{-1 / 2}$ and so always appears in the dimensionless combination $g \hbar^{1 / 2}$. This implies that our loop expansion is an expansion in $g^{2} \hbar$. We will set $\hbar=1$.
Consider two adjacent minima $y_{1}$ and $y_{2}>y_{1}$ of the potential $V$ such that

$$
V\left(y_{1}+y\right)=V\left(y_{2}-y\right) \quad \text { if } y \in\left[0, y_{2}-y_{1}\right] .
$$

For convenience, the potential may always be shifted so that $V\left(y_{1}\right)=0$.

Define the shifted field

$$
\tilde{\phi}(x)=\phi(x)-\frac{y_{1}}{g} .
$$

Note that the shifted field is canonically conjugate to the original $\pi(x)$. Expanding the Hamiltonian to second order in $\tilde{\phi}$, one sees that it is a scalar field of mass squared

$$
m^{2}=\left.M^{2} \partial_{y}^{2} V(y)\right|_{y=y_{1}}
$$

In the Schrödinger picture, the shifted field and its conjugate $\pi(x)$ can be decomposed as usual into oscillator modes $a_{p}^{\dagger}$ and $a_{p}$ which satisfy a Heisenberg algebra

$$
\begin{aligned}
& \tilde{\phi}(x)=\int \frac{d p}{2 \pi} \frac{1}{\sqrt{2 \omega_{p}}}\left(a_{p}^{\dagger}+a_{-p}\right) e^{-i p x}, \\
& \pi(x)=i \int \frac{d p}{2 \pi} \frac{\sqrt{\omega_{p}}}{\sqrt{2}}\left(a_{p}^{\dagger}-a_{-p}\right) e^{-i p x},
\end{aligned}
$$

where

$$
\omega_{p}=\sqrt{m^{2}+p^{2}} .
$$

The normal ordering is defined with respect to these modes. Recall that, in $(1+1)$-dimensional scalar field theories with such nonderivative potentials, normal ordering is sufficient to render the theory finite in the ultraviolet. In a sense, the normal ordering corresponds to adding a certain choice of counterterm after which regularization is no longer required. We claim that the calculation below would proceed similarly with a more general counterterm and regulator and so can also be applied to more interesting field theories. However, the proof of this claim will be left to future work.

The classical field theory admits a stationary kink solution $\tilde{\phi}(x, t)=f(x)$ which solves

$$
f^{\prime \prime}(x)=\frac{M^{2}}{g} V^{\prime}\left(g f(x)+y_{1}\right)
$$

with boundary conditions $f(-\infty)=0$ and $f(+\infty)=$ $y_{2}-y_{1}$. If $f_{1}(x)$ is the solution that would be obtained at $g=1$, then $f(x)=f_{1}(x) / g$. In this sense, $f$ is of the order of $O(1 / g)$. 
Let us define the displacement operator

$$
\mathcal{D}_{f}=\exp \left(-i \int d x f(x) \pi(x)\right),
$$

which satisfies the relation [11]

$: F(\pi(x), \tilde{\phi}(x)): \mathcal{D}_{f}=\mathcal{D}_{f}: F(\pi(x), \tilde{\phi}(x)+f(x)):$

and is unitary. It defines the new Hamiltonian $H_{K}$ via the definition

$$
H \mathcal{D}_{f}=\mathcal{D}_{f} H_{K}
$$

The key observation behind this paper is that Eq. (12) can be used to uniquely define the operator $H_{K}$ however $H$ is regulated and whatever counterterms have been added to $H$. This provides the matching prescription advertised above. In the case at hand,

$H_{K}=H_{0}+\int d x:\left[\frac{M^{2}}{g^{2}} V\left(g \tilde{\phi}(x)+g f(x)+y_{1}\right)\right]:$.

If $V$ is analytic at $y_{1}$, we may expand $V$ and so $H_{K}$ in a power series in $\tilde{\phi}$. All terms of order $\tilde{\phi}^{k}$ are multiplied by $g^{k-2}$. Thus, the cubic terms and above may be treated in perturbation theory in $g$. At the order of $O\left(g^{0}\right)$, we find the truncated Hamiltonian $H_{K 0}$ :

$$
\begin{aligned}
H_{K} & =H_{K 0}+E_{\mathrm{cl}}+O(g), \\
E_{\mathrm{cl}} & =\int d x \frac{f^{\prime}(x)^{2}}{2}+\frac{M^{2}}{g^{2}} V\left(f_{1}(x)+y_{1}\right), \\
H_{K 0} & =H_{0}+\frac{M^{2}}{2} \int d x V^{\prime \prime}\left(f_{1}(x)+y_{1}\right): \tilde{\phi}(x)^{2}:
\end{aligned}
$$

Here, $E_{\mathrm{cl}}$ is the classical kink mass. By dimensional analysis, $E_{\mathrm{cl}}$ is of the order of $O\left(M / g^{2}\right)$. Therefore, the $g$-independent correction that one may expect from $H_{K 0}$ is suppressed by $g^{2} \hbar$ with respect to $E_{\mathrm{cl}}$ and so is the one-loop correction. The $O(g)$ corrections on the first line then enter only at two loops and beyond. What is $H_{K 0}$ ?

The energy of a state is its eigenvalue with respect to the Hamiltonian $H$. Let us consider two eigenstates of $H$, the vacuum $|0\rangle$ with $\langle 0|\tilde{\phi}(x)| 0\rangle=0$ and the ground state of the kink sector, hereafter called the kink state $|K\rangle$. The mass $M_{K}$ of the kink is just the difference between their two eigenvalues:

$E_{0}|0\rangle=H|0\rangle, \quad E_{K}|K\rangle=H|K\rangle, \quad M_{K}=E_{K}-E_{0}$.

Note that the same operator $H$ appears in each expression, so there is only one operator to regularize and so no need to match regulators.
Following the logic of Ref. [12], $\mathcal{D}_{f}|0\rangle$ is in the one-kink sector because $\left\langle 0\left|\mathcal{D}_{f}^{-1} \tilde{\phi}(x) \mathcal{D}_{f}\right| 0\right\rangle=f(x)$. However, it is not the kink ground state $|K\rangle$; indeed, it is not even an eigenstate of the Hamiltonian. As argued in Ref. [13], the kink ground state will contain loop corrections to this naive formula. We will encode these corrections in an operator $\mathcal{O}$ defined to be any operator which satisfies

$$
|K\rangle=\mathcal{D}_{f} \mathcal{O}|0\rangle .
$$

Then, using Eq. (15),

$E_{K} \mathcal{O}|0\rangle=\mathcal{D}_{f}^{-1} E_{K} \mathcal{D}_{f} \mathcal{O}|0\rangle=\mathcal{D}_{f}^{-1} H \mathcal{D}_{f} \mathcal{O}|0\rangle=H_{K} \mathcal{O}|0\rangle$.

That $E_{K}$ is an eigenvalue of $H_{K}$ is obvious, as $H_{K}$ and $H$ are related by a similarity transformation and so have the same eigenvalues. The lowest eigenstate of $H_{K}$ is $\mathcal{D}_{f}^{-1} \mathcal{O}|0\rangle$, which has eigenvalue $E_{0}$.

$\mathcal{O}$ can be taken to be equal to the identity plus perturbative loop corrections. This means that one may truncate $H_{K}$ to any desired order in $g$ and find $\mathcal{O}$ and $E_{K}$ at the same order. Inserting Eq. (14) expanded to the order of $O\left(g^{0}\right)$, Eq. (17) becomes the eigenvalue problem

$$
H_{K 0} \mathcal{O}|0\rangle=\left(E_{K}-E_{\mathrm{cl}}\right) \mathcal{O}|0\rangle,
$$

which can be solved by diagonalizing $H_{K 0}$. The free Hamiltonian $H_{K 0}$ describes the linearized theory of local perturbations to the kink, and so its eigenstates are all in the one-kink sector. Thus, the lowest-energy eigenstate of $H_{K 0}$ is the kink ground state $\mathcal{O}|0\rangle$. To find it, we will now diagonalize $H_{K 0}$.

At leading order, the classical equation of motion derived from $H_{K 0}$ is

$-\partial_{t}^{2} F(x, t)+\partial_{x}^{2} F(x, t)=M^{2} V^{\prime \prime}\left(g f(x)+y_{1}\right) F(x, t)$.

Decomposing $F(x, t)=F(x) e^{-i \omega t}$, one obtains

$$
\partial_{x}^{2} F(x)=\left(-\omega^{2}+M^{2} V^{\prime \prime}\left(g f(x)+y_{1}\right)\right) F(x) .
$$

We will be interested in such solutions which remain bounded as $x \rightarrow \pm \infty$. Defining

$$
V_{I}(\tilde{\phi})=V(\tilde{\phi})-\frac{m^{2}}{2} \tilde{\phi}^{2} \quad k^{2}=\omega^{2}-m^{2},
$$

this becomes

$$
\partial_{x}^{2} F(x)=\left(-k^{2}+M^{2} V_{I}^{\prime \prime}\left(g f(x)+y_{1}\right)\right) F(x) .
$$

There will be continuum solutions and also $\beta+1$ bound state solutions, which we will index by $\mu \in[0, \beta]$ with $\mu=0$ corresponding to the Goldstone mode. Because of 
the condition (4), the bound state solutions $F_{B, \mu}$ will be even or odd, while the continuum solutions $F_{k}$ at each $k$ can be decomposed into an even and odd part. We will organize all solutions $F(x)$, bound or unbound, such that the real part is even and the imaginary part is odd $F^{*}(x)=F(-x)$, and we will normalize them so that

$$
\begin{aligned}
\int d x F_{k}(x) F_{l}^{*}(x) d x & =2 \pi \delta(k-l), \\
\int d x F_{B, \mu}(x) F_{B, \nu}^{*}(x) d x & =\delta_{\mu \nu .}
\end{aligned}
$$

Next, we decompose $\tilde{\phi}$ and $\pi$ into contributions from the continuum and bound states:

$$
\begin{aligned}
& \phi(x)=\phi_{C}(x)+\sum_{\mu=0}^{\beta} \phi_{B, \mu}(x), \\
& \pi(x)=\pi_{C}(x)+\sum_{\mu=0}^{\beta} \pi_{B, \mu}(x),
\end{aligned}
$$

which, in turn, are decomposed in terms of solutions:

$$
\begin{aligned}
\phi_{C}(x) & =\int \frac{d k}{2 \pi} \frac{1}{\sqrt{2 \omega_{k}}}\left(b_{k}^{\dagger}+b_{-k}\right) F_{k}(x), \\
\phi_{B, i}(x) & =\frac{1}{\sqrt{2 \omega_{B, i}}}\left(b_{B, i}^{\dagger}-P_{i} b_{B, i}\right) F_{B, i}(x), \\
\phi_{B, 0}(x) & =\phi_{0} F_{b, 0}(x), \pi_{B, 0}(x)=\pi_{0} F_{B, 0}(x), \\
\pi_{C}(x) & =i \int \frac{d k}{2 \pi} \sqrt{\frac{\omega_{k}}{2}}\left(b_{k}^{\dagger}-b_{-k}\right) F_{k}(x), \\
\pi_{B, i}(x) & =i \sqrt{\frac{\omega_{B, i}}{2}}\left(b_{B, i}^{\dagger}+P_{i} b_{B, i}\right) F_{B, i}(x),
\end{aligned}
$$

where the index $i \in[1, \beta]$ runs over all bound states except for the Goldstone mode $\mu=0$, which has frequency $\omega_{B, 0}=0$ and the sign $P_{i}$ is the parity of the $i$ th bound state.

Combining Eqs. (7) and (24), one can find the Bogoliubov transformation relating the two sets of oscillators:

$$
\begin{aligned}
a_{p} & =a_{C, p}+\sum_{\mu=0}^{\beta} a_{B, \mu, p}, \\
a_{C, p} & =\int \frac{d k}{2 \pi} \frac{\hat{F}_{k}^{*}(p)}{2}\left(\frac{\omega_{p}-\omega_{k}}{\sqrt{\omega_{p} \omega_{k}}} b_{k}^{\dagger}+\frac{\omega_{p}+\omega_{k}}{\sqrt{\omega_{p} \omega_{k}}} b_{k}\right), \\
a_{B, i, p} & =\frac{\hat{F}_{B, i}^{*}(p)}{2}\left(\frac{\omega_{p}-\omega_{B, i}}{\sqrt{\omega_{p} \omega_{B, i}}} b_{B, i}^{\dagger}+P_{i} \frac{\omega_{p}+\omega_{B, i}}{\sqrt{\omega_{p} \omega_{B, i}}} b_{B, i}\right), \\
a_{B, 0, p} & =\hat{F}_{B, 0}^{*}(p)\left[\sqrt{\frac{\omega_{p}}{2}} \phi_{0}+\frac{i}{\sqrt{2 \omega_{p}}} \pi_{0}\right],
\end{aligned}
$$

where $\hat{F}$ is the Fourier transform of $F$. This Fourier transform will generally contain a term proportional to $\delta(k-p)$ and, if the potential in $H_{K 0}$ is not reflectionless, also $\delta(k+p)$. These delta functions can be used to evaluate the $k$ integrals in their respective terms. As $\omega_{p}=\omega_{-p}$, one sees from Eq. (25) that these terms do not lead to mixing between $a$ and $b^{\dagger}$. They therefore preserve the normal ordering and so will not contribute to soliton masses.

Recall that $H_{K 0}$ in Eq. (14) is normal ordered in terms of $a$ and $a^{\dagger}$, so that all $a^{\dagger}$ appear on the left. Inserting Eq. (25), it may be written in terms of $b^{\dagger}$ and $b$. By direct computation, one can see that there are no $b^{\dagger} b^{\dagger}$ or $b b$ terms. However, there will be terms of the form $b b^{\dagger}$. By inverting Eq. (24) and using the canonical commutation relations of $\tilde{\phi}$ and $\pi$, one finds that $b$ satisfy the algebra

$$
\begin{aligned}
{\left[b_{k_{1}}, b_{k_{2}}^{\dagger}\right] } & =2 \pi \delta\left(k_{1}-k_{2}\right), \\
{\left[b_{B, i}, b_{B, j}^{\dagger}\right] } & =\delta_{i j}, \quad\left[\phi_{0}, \pi_{0}\right]=i .
\end{aligned}
$$

Using these commutation relations and the defining equation (22) of $F, H_{K O}$ may be reordered to place all $b^{\dagger}$ on the left, yielding

$$
\begin{aligned}
H_{K 0}= & \int \frac{d k}{2 \pi} \omega_{k} b_{k}^{\dagger} b_{k}+\sum_{i} \omega_{B, i} b_{B, i}^{\dagger} b_{B, i}+\frac{\pi_{0}^{2}}{2}+Q, \\
Q= & -\frac{1}{4}\left[\int \frac{d k}{2 \pi} \int \frac{d p}{2 \pi} \frac{\left(\omega_{p}-\omega_{k}\right)^{2}}{\omega_{p}} \hat{F}_{k}^{2}(p)\right. \\
& \left.+\sum_{\mu=0}^{\beta} \int \frac{d p}{2 \pi} \frac{\left(\omega_{p}-\omega_{B, \mu}\right)^{2}}{\omega_{p}} \hat{F}_{B, \mu}^{2}(p)\right] .
\end{aligned}
$$

This is just a sum of harmonic oscillators.

Therefore, the lowest-energy mode, $\mathcal{O}|0\rangle$, is the one annihilated by all $b$ :

$$
b_{k} \mathcal{O}|0\rangle=b_{B, i} \mathcal{O}|0\rangle=\pi_{0} \mathcal{O}|0\rangle=0,
$$

and its corresponding energy is $E_{K}=E_{\mathrm{cl}}+Q$. The oneloop correction to the kink mass is just $Q$. In Refs. [11,14], we show that, in the case of the $\phi^{4}$ kink and sine-Gordon soliton, this agrees with the one-loop result obtained using mode matching but not that obtained using an energy cutoff.

This is our main result. However, by diagonalizing the Hamiltonian, we have obtained the entire spectrum, at one loop. It consists of harmonic oscillator spectra excited with various $b^{\dagger}$ and also rigid momenta $\pi_{0}$. To calculate the higher-loop corrections is now straightforward. One need only consider the full Hamiltonian in Eq. (14) and use ordinary perturbation theory in $g^{2}$, in terms of the $b$ and $b^{\dagger}$ oscillators. At two loops, there will also be corrections to the kink mass from the one-loop correction to the vacuum energy, which can also be calculated in standard perturbation theory as in Ref. [15]. These higher-loop corrections will be reported in future work. 
Our long-term goal is to find the monopole operator responsible for confinement in Yang-Mills theory and to show that it is tachyonic as suggested by the paradigm of Refs. [16,17]. To arrive there, we will try to extend our construction to $\mathcal{N}=2$ super Yang-Mills, where there is a continuous deformation from the semiclassical to the condensing monopole [18]. Here, we will start by reproducing the one-loop corrections already found in Ref. [19].

\section{ACKNOWLEDGMENTS}

J.E. is supported by the Chinese Academy of Sciences Key Research Program of Frontier Sciences Grant No. QYZDY-SSW-SLH006 and the National Science Foundation of China MianShang Grants No. 11875296 and No. 11675223. J.E. also thanks the Recruitment Program of High-end Foreign Experts for support.
[1] A. Rebhan and P. van Nieuwenhuizen, No saturation of the quantum Bogomolnyi bound by two-dimensional supersymmetric solitons, Nucl. Phys. B508, 449 (1997).

[2] R. F. Dashen, B. Hasslacher, and A. Neveu, Nonperturbative Methods and extended hadron models in field theory 2. Two-dimensional models and extended hadrons, Phys. Rev. D 10, 4130 (1974).

[3] R. Rajaraman, Some nonperturbative semiclassical methods in quantum field theory: A pedagogical review, Phys. Rep. 21, 227 (1975).

[4] A. S. Goldhaber, A. Rebhan, P. van Nieuwenhuizen, and R. Wimmer, Quantum corrections to mass and central charge of supersymmetric solitons, Phys. Rep. 398, 179 (2004).

[5] H. Nastase, M. A. Stephanov, P. van Nieuwenhuizen, and A. Rebhan, Topological boundary conditions, the BPS bound, and elimination of ambiguities in the quantum mass of solitons, Nucl. Phys. B542, 471 (1999).

[6] A. S. Goldhaber, A. Litvintsev, and P. van Nieuwenhuizen, Local Casimir energy for solitons, Phys. Rev. D 67, 105021 (2003).

[7] R. Wimmer, Quantization of supersymmetric solitons, arXiv:hep-th/0109119

[8] J. F. Schonfeld, Soliton masses in supersymmetric theories, Nucl. Phys. B161, 125 (1979).

[9] A. Alonso Izquierdo, W. Garcia Fuertes, M. A. Gonzalez Leon, M. de la Torre Mayado, J. Mateos Guilarte, and J. M. Munoz Castaneda, Lectures on the mass of topological solitons, arXiv:hep-th/0611180.
[10] A. Rebhan, P. van Nieuwenhuizen, and R. Wimmer, Quantum Corrections to Solitons and BPS Saturation, Fundamental Interactions (World Scientific, Singapore, 2009), pp. 41-74, https://doi.org/10.1142/9789814277839_ 0005.

[11] J. Evslin, Manifestly finite derivation of the quantum kink mass, J. High Energy Phys. 11 (2019) 161.

[12] K. Hepp, The classical limit for quantum mechanical correlation functions, Commun. Math. Phys. 35, 265 (1974).

[13] J. G. Taylor, Solitons as infinite constituent bound states, Ann. Phys. (N.Y.) 115, 153 (1978).

[14] H. Guo and J. Evslin, Finite derivation of the one-loop sineGordon soliton mass, J. High Energy Phys. 02 (2020) 140.

[15] H. Liu, Y. Zhou, and J. Evslin, Ground states of the $\phi^{4}$ double-well QFT, arXiv:1909.04946.

[16] G. 't Hooft, Topology of the gauge condition and new confinement phases in nonabelian gauge theories, Nucl. Phys. B190, 455 (1981).

[17] S. Mandelstam, vortices and quark confinement in nonabelian gauge theories, Phys. Rep. 23, 245 (1976).

[18] N. Seiberg and E. Witten, Electric-magnetic duality, monopole condensation, and confinement in $N=2$ supersymmetric Yang-Mills theory, Nucl. Phys. B426, 19 (1994); Erratum, Nucl. Phys. B430, 485 (1994).

[19] A. Rebhan, P. van Nieuwenhuizen, and R. Wimmer, A new anomaly in the central charge of the $N=2$ monopole, Phys. Lett. B 594, 234 (2004). 